\title{
On Some Homological Properties of Hypergroup Algebras with Relation to Their Character Spaces
}

\author{
Amir Sahami $\mathbb{D}^{1}{ }^{1}$ Mehdi Rostami, ${ }^{2}$ Seyedeh Fatemeh Shariati, ${ }^{2}$ and Salman Babayi ${ }^{3}$ \\ ${ }^{1}$ Department of Mathematics Faculty of Basic Science, Ilam University, P.O. Box 69315-516, Ilam, Iran \\ ${ }^{2}$ Department of Mathematics and Computer Science, Amirkabir University of Technology (Tehran Polytechnic), Tehran, Iran \\ ${ }^{3}$ Department of Mathematics, Faculty of Science, Urmia University, Urmia, Iran
}

Correspondence should be addressed to Amir Sahami; a.sahami@ilam.ac.ir

Received 25 October 2021; Revised 8 December 2021; Accepted 6 January 2022; Published 29 January 2022

Academic Editor: Ralf Meyer

Copyright ( $) 2022$ Amir Sahami et al. This is an open access article distributed under the Creative Commons Attribution License, which permits unrestricted use, distribution, and reproduction in any medium, provided the original work is properly cited.

In this paper, we study the notion of approximate biprojectivity and left $\varphi$-biprojectivity of some Banach algebras, where $\varphi$ is a character. Indeed, we show that approximate biprojectivity of the hypergroup algebra $L^{1}(K)$ implies that $K$ is compact. Moreover, we investigate left $\varphi$-biprojectivity of certain hypergroup algebras, namely, abstract Segal algebras. As a main result, we conclude that (with some mild conditions) the abstract Segal algebra $B$ is left $\varphi$-biprojective if and only if $K$ is compact, where $K$ is a hypergroup. We also study the approximate biflatness and left $\varphi$-biflatness of hypergroup algebras in terms of amenability of their related hypergroups.

\section{Introduction and Preliminaries}

Hypergroups are a suitable generalization of classical locally compact groups. In classical setting, the convolution of two point mass measures is a point mass measure, while in hypergroup structure, the convolution of two point mass measures is a probability measure with compact support. The study of hypergroups was initiated in the 1970 s by Dunkl [1], Jewwet [2], and Spector [3], each of them in various axioms. However, in this paper, we will base our work on Jewett's axioms in [2].

Biprojectivity is an important homological notion that arises naturally in Helemskii's works in the 1980s; interested readers can refer to his comprehensive book [4]. Biprojectivity of some well-known Banach algebras associated to locally compact groups, such as group algebras and measure algebras, is studied in $[4,5]$. Biprojectivity of the hypergroup algebra $L^{1}(K)$ is studied in [6]. As a generalization of this notion, Y. Zhang in [7] introduced the notion of approximate biprojectivity. Indeed, a Banach algebra $A$ is called approximately biprojective if there exists a net $\left(\rho_{\alpha}\right)$ of continuous $A$-bimodule morphism from $A$ into $A^{\wedge} \otimes A$ such that $\pi_{A}{ }^{\circ} \rho_{\alpha}(\mathrm{a}) \longrightarrow \mathrm{a}$ for every $a \in A$, where
$\pi_{A}: A^{\wedge} \otimes A \longrightarrow A$ is the diagonal operator defined by $\pi_{A}(a \otimes b)=a b$. For recent works about this concept, refer to [8].

Throughout the paper, $\Delta(A)$ stands for the set of all nonzero multiplicative linear functionals on $A$. Kaniuth et al. [9] introduced the notion of left $\varphi$-amenable Banach algebras $(\varphi \in \Delta(A))$ as a generalization of the notion of amenable Banach algebras introduced by Johnson in [10]. A Banach algebra $A$ is called left $\varphi$-amenable if every derivation $D$ from $A$ into $X^{*}$ is inner, for every Banach $A$-bimodule $X$ with the left module action $a \cdot x=\varphi(a) x$ for all $a \in A$ and $x \in X$.

$\mathrm{Hu}$ et al. in [11] defined the notion of left $\varphi$-contractibility for Banach algebras. Following [12], a Banach algebra $A$ is called left $\varphi$-contractible, where $\varphi \in \Delta(A)$, if there exists $\mathbf{m} \in A$ such that $a \mathbf{m}=\varphi(a) \mathbf{m}$ and $\varphi(\mathbf{m})=1$, for every $a \in A$. For a locally compact group $G$, it is shown that left $\varphi$-contractibility of $L^{1}(G)$ (or $M(G)$ ) is equivalent to compactness of $G$ (Theorem 6.1 in [15]).

Motivated by these considerations, the first author defined the homological notion of left $\varphi$-biprojectivity for Banach algebras (see, e.g., [13]). Here is the definition of his new notion. A Banach algebra $A$ is called left $\varphi$-biprojective, 
where $\varphi \in \Delta(A)$, if there exists a bounded linear map $\rho: A \longrightarrow A^{\wedge} \otimes A$ such that

$$
\begin{aligned}
\rho(a b) & =a \cdot \rho(b)=\varphi(b) \rho(a), \\
\varphi^{\circ} \pi_{A}{ }^{\circ} \rho(a) & =\varphi(a), \quad(a, b \in A) .
\end{aligned}
$$

The right case can be similarly defined.

The first and the second authors in [13] explained the relation between left $\varphi$-contractibility and left $\varphi$-biprojectivity. They proved that left $\varphi$-contractibility implies left $\varphi$-biprojectivity, and the converse is valid if $A$ is commutative or has a left approximate identity.

We give some brief backgrounds on hypergroups and their related algebras and establish our notation; for details, see [14]. Let $K$ be a locally compact Hausdorff space and $M(K)$ denote the set of all bounded complex Radon measures on $K$, where the norm of each measure $\mu \in M(K)$ is the total variation $|\mu|$. Also, we denote by $\mathscr{C}(K)$ the set of all nonempty compact subsets of $K$ and equipped this space with the Michael topology, that is, the topology generated by the sets

$$
U_{V, W}:=\{L \in \mathscr{C}(K): L \cap V \neq \varnothing, \quad L \subseteq W\},
$$

for open subsets $V$ and $W$ in $K$.

The space $K$ is called a hypergroup if there is a convolution $*: M(K) \times M(K) \longrightarrow M(K)$, an involution $x \mapsto \bar{x}$ on $K$, and an element $e \in K$ (called the identity element) such that the following holds:

(i) $(M(K), *)$ is a Banach algebra.

(ii) $\delta_{x} * \delta_{y}$ is a probability measure with compact support.

(iii) The map $(x, y) \mapsto \delta_{x} * \delta_{y}$ is continuous from $K \times K$ into $M(K)$ equipped with the weak * topology.

(iv) The map $(x, y) \mapsto \operatorname{supp}\left(\delta_{x} * \delta_{y}\right)$ is continuous from $K \times K$ into $\mathscr{C}(K)$.

(v) For each $x \in K, \delta_{x} * \delta_{e}=\delta_{e} * \delta_{x}=\delta_{x}$.

(vi) The mapping $x \mapsto \bar{x}$ is a homeomorphism on $K$ of period 2 and $\left(\delta_{x} * \delta_{y}\right)^{-}=\delta_{\bar{y}} * \delta_{\bar{x}} \quad$ and $e \in \operatorname{supp}\left(\delta_{x} * \delta_{y}\right)$ if and only if $x=\bar{y}$ for each $x, y \in K$. Here, the measure $\mu^{-}$is given by $\mu^{-}(E)=$ $\mu(\bar{E})$ for all Borel subsets $E \subseteq K$.

A hypergroup $K$ is called commutative if $\delta_{x} * \delta_{y}=\delta_{y} * \delta_{x}$ for all $x, y \in K$. In $[2,3]$, it is proved that every commutative or compact hypergroup has a unique (left) Haar measure. The existence and the uniqueness of left Haar measure on a general locally compact hypergroup were proved recently by Chapovsky in [15]. Throughout this paper, we assume that $K$ is a hypergroup with a unique Haar measure $\omega_{K}$. That is,

$$
\int_{k} f(x * y) d \omega_{k}(y)=\int_{K} f(y) d \omega_{K}(y), \quad(x \in K),
$$

for every Borel measurable function $f$ on $K$. Then, $\left(L^{1}(K),\|\cdot\|_{1}\right)$ with the involution $f^{*}(x)=\overline{f(\bar{x})}$ and the convolution

$$
f * g(x)=\int_{K} f(y) g(\bar{y} * x) d \omega_{K}(y),
$$

is a Banach $*$-algebra, where $f(x * y)=\int_{K} f d\left(\delta_{x} * \delta_{y}\right)$ for every $x, y \in K$. A nonzero bounded continuous function $\chi: K \longrightarrow \mathbb{C}$ is called a character on $K$ if $\chi(x * y)=\chi(x) \chi(y)$ for every $x, y \in K$. The set of all characters on $K$ will be denoted by $\Upsilon_{b}(K)$, i.e.,

$\Upsilon_{b}(K)=\left\{\chi \in C_{b}(K): \chi \neq 0, \chi(x * y)=\chi(x) \chi(y) \quad(x, y \in K)\right\}$.

For $\chi \in \Upsilon_{b}(K)$, define $\varphi_{\chi}$ on $L^{1}(K)$ via

$$
\varphi_{\chi}(f)=\int_{K} \overline{\chi(s)} f(s) \mathrm{d} \omega_{K}(s) .
$$

It may be observed that $\varphi_{\chi} \in \Delta\left(L^{1}(K)\right)$, and note that there is at least one character on $L^{1}(K)$, namely, the augmentation character $\varphi_{1}$. Further, if $K$ is commutative, it is well-known that there is no other character on $L^{1}(K)$; that is, $\Delta\left(L^{1}(K)\right)=\left\{\varphi_{\chi}: \chi \in \Upsilon_{b}(K)\right\}$. For more details, see Section 2.2 in [3].

In the present paper, we show that approximate biprojectivity of hypergroup algebra $L^{1}(K)$ implies that $K$ is compact. After that, we study left $\varphi$-biprojectivity of general abstract Segal algebras with respect to the hypergroup algebra $L^{1}(K)$. As a result (with a mild condition), we conclude that the abstract Segal algebra $B$ is left $\varphi$-biprojective if and only if $K$ is compact. We also study approximate biflatness and left $\phi$-biflatness of some hypergroup algebras in terms of amenability of their related hypergroups.

\section{Approximate Biprojectivity and Left $\varphi$-Biprojectivity of Hypergroup Algebras}

Recall that if $A$ is a Banach algebra and $I$ is a closed two-sided ideal in $\mathscr{A}$, then for each $\varphi \in \Delta(A)$ such that $I \subseteq \operatorname{ker} \varphi$, the map $\bar{\varphi}(a+I)=\varphi(a)$ is a character on $(A / I)$.

Proposition 1. Let $A$ be a Banach algebra, $\varphi \in \Delta(A)$, and let $I \subseteq k e r \varphi$ be a closed two-sided ideal of $A$ such that $\overline{A I}=I$. If $A$ is left $\varphi$-biprojective, then $(A / I)$ is left $\bar{\varphi}$-biprojective.

Proof. Since $A$ is left $\varphi$-biprojective, there is a bounded linear map $\rho: A \longrightarrow A^{\wedge} \otimes A$ such that $a \cdot \rho(b)=\rho(a b)=\varphi(b) \rho(a)$ and $\varphi^{\circ} \pi_{A}{ }^{\circ} \rho(\mathrm{a})=\varphi(\mathrm{a})$ for every $a \in A$. Consider the quotient map $q: A \longrightarrow(A / I)$. It may be noted that

$$
\left(i d_{A} \otimes q\right)^{\circ} \rho(\mathrm{at})=\mathrm{id}_{\mathrm{A}} \otimes \mathrm{q}(\varphi(\mathrm{t}) \rho(\mathrm{a}))=0 \quad(\mathrm{a} \in \mathrm{A}, \mathrm{t} \in \mathrm{I}) .
$$

By the assumption $\overline{A I}=I$, we have $\left.\left(i d_{A} \otimes q\right)^{\circ} \rho\right|_{I}=0$. Thus, $\left(i d_{A} \otimes q\right)^{\circ} \rho$ can be dropped on $(A / I)$. Define $\eta:(A / I) \longrightarrow(A / I)^{\wedge} \otimes(A / I)$ $\eta=\left(q \otimes i d_{(A / I)}\right)^{\circ}\left(\mathrm{id}_{\mathrm{A}} \otimes \mathrm{q}\right)^{\circ} \rho$. It may be observed that

$$
\bar{\varphi}^{\circ} \pi_{(\mathrm{A} / \mathrm{I})}{ }^{\circ} \eta(\mathrm{a}+\mathrm{I})=\varphi(\mathrm{a})=\bar{\varphi}(\mathrm{a}+\mathrm{I}) \quad(\mathrm{a} \in \mathrm{A}) .
$$


Moreover,

$$
\begin{aligned}
\eta((a+I)(b+I)) & =\left(q \otimes i d_{(A / I)}\right) \circ\left(\mathrm{id}_{\mathrm{A}} \otimes \mathrm{q}\right)^{\circ} \rho(\mathrm{ab}) \\
& =\left(q \otimes i d_{(A / I)}\right)^{\circ}\left(\mathrm{id}_{\mathrm{A}} \otimes \mathrm{q}\right)^{\circ}(\mathrm{a} \cdot \rho(\mathrm{b})) \\
& =a \cdot\left(q \otimes i d_{(A / I)}\right)^{\circ}\left(\mathrm{id}_{\mathrm{A}} \otimes \mathrm{q}\right)^{\circ} \rho(\mathrm{b}) \\
& =(a+I) \cdot \eta(b+I),
\end{aligned}
$$

and also

$$
\begin{aligned}
\eta((a+I)(b+I)) & =\left(q \otimes i d_{(A / I)}\right) \circ\left(\mathrm{id}_{\mathrm{A}} \otimes \mathrm{q}\right)^{\circ} \rho(\mathrm{ab}) \\
& =\left(q \otimes i d_{(A / I)}\right) \circ\left(\mathrm{id}_{\mathrm{A}} \otimes \mathrm{q}\right)^{\circ}(\varphi(\mathrm{b}) \rho(\mathrm{a})) \\
& =a \cdot\left(q \otimes i d_{(\mathrm{A} / I)}\right)^{\circ}\left(\mathrm{id}_{\mathrm{A}} \otimes \mathrm{q}\right)^{\circ} \rho(\mathrm{a}) \\
& =\bar{\varphi}(b+I) \eta(a+I) .
\end{aligned}
$$

Hence, $(A / I)$ is left $\bar{\varphi}$-biprojective.

Corollary 1. Let $A$ be a Banach algebra with a left ap-

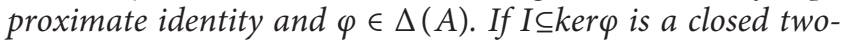
sided ideal of $A$ and $A$ is left $\varphi$-biprojective, then $(A / I)$ is left $\bar{\varphi}$-biprojective.

In the group case, it is well-known that the group algebra $L^{1}(K)$ is biprojective if and only if $G$ is compact (see, for example, [4]). In the similar way, in Theorem 3.1 in [6], it is shown that if the hypergroup algebra $L^{1}(K)$ is biprojective, then $K$ is compact. In the following, we give a generalization of this result and characterize approximate biprojectivity of the hypergroup algebra $L^{1}(K)$.

Theorem 1. Let $K$ be a locally compact hypergroup. If the hypergroup algebra $L^{1}(K)$ is approximately biprojective, then $K$ is compact.

Proof. Let $L^{1}(K)$ be approximately biprojective. Since $L^{1}(K)$ has a bounded approximate identity (Theorem 1.6.15 in [3]), the hypergroup algebra $L^{1}(K)$ is left $\varphi$-contractible for every $\varphi \in \Delta\left(L^{1}(K)\right)$ (Theorem 3.9 in [20]). Consider the augmentation character on $L^{1}(K)$ via

$$
\varphi_{1}(f)=\int_{K} f \mathrm{~d} \omega_{K} .
$$

So, there exists $\mathbf{m} \in L^{1}(K)$ satisfying $f * \mathbf{m}=\varphi_{1}(f) \mathbf{m}$ and $\varphi_{1}(\mathbf{m})=1$ for every $f \in L^{1}(K)$. Choose $f \in L^{1}(K)$ such that $\varphi_{1}(f)=1$. It is worth noting that

$$
\begin{aligned}
\delta_{x} * \mathbf{m} & =\varphi_{1}(f) \delta_{x} * \mathbf{m}, \\
& =\delta_{x} *\left(\varphi_{1}(f) \mathbf{m}\right), \\
& =\delta_{x} *(f * \mathbf{m}), \\
& =\left(\delta_{x} * f\right) * \mathbf{m}, \\
& =\varphi_{1}\left(\delta_{x} * f\right) \mathbf{m} .
\end{aligned}
$$

Since $\delta_{\bar{x}} * \omega_{K}=\omega_{K}$, we have $\varphi_{1}\left(\delta_{x} * f\right)=\varphi_{1}(f)$. Thus, $\delta_{x} * \mathbf{m}=\mathbf{m}$. It follows that $\mathbf{m}$ is a constant function. Therefore, Tex translation failed. The latter implies that $K$ is compact (page 40 in [3]).
We study left $\phi$-biprojectivity of abstract Segal algebras. So, we start with definition of abstract Segal algebras.

Definition 1. Let $A$ be a Banach algebra with norm $\|\cdot\|_{A}$. We say that a Banach algebra $B$ with norm $\|\cdot\|_{B}$ is an abstract Segal algebra with respect to $A$ if

(i) $B$ is a dense left ideal in $\mathrm{A}$

(ii) There exists $M>0$ such that $\|b\|_{A} \leq M\|b\|_{B}$ for every $b \in B$

(iii) There exists $C>0$ such that $\|a b\|_{B} \leq C\|a\|_{A}\|b\|_{B}$ for every $a \in A$ and $b \in B$

Moreover, we say that $B$ is a symmetric abstract Segal algebras if $B$ is a two-sided ideal of $A$ and there exists $C>0$ such that $\|b a\|_{B} \leq C\|a\|_{A}\|b\|_{B}$ for every $a \in A$ and $b \in B$.

For more details about abstract Segal algebras, see [16]. We note that there are some abstract Segal algebras which are not symmetric. Homological and cohomological properties of abstract Segal algebras have been studied in many papers (see, for example, [17-19]). Recall that it is known that $\Delta(B)=\left\{\left.\varphi\right|_{B}: \varphi \in \Delta(A)\right\}$ (see Lemma 2.2 in [1]). It is worthwhile to mention that Essmaili et al. in [6] studied right $\varphi$-biprojectivity (they called it condition $Q$ ) of Banach algebras associated with a hypergroup, especially symmetric Segal algebras. As a main result, they showed that if $K$ is a commutative hypergroup and $B$ is a symmetric abstract Segal algebra with respect to $L^{1}(K)$, then $B$ is right $\left.\varphi\right|_{B}$-biprojective if and only if $K$ is compact, where $\varphi \in \Delta\left(L^{1}(K)\right)$. In the following, using Theorem 1, we extend the left version of (Corollary 3.11 in [6]).

Theorem 2. Let $K$ be a locally compact hypergroup, $\varphi_{\chi} \in \Delta\left(L^{1}(K)\right)$, and let $B$ be an abstract Segal algebra with respect to $L^{1}(K)$ which possess a left approximate identity. Then, the following statements are equivalent:

\section{(i) $B$ is left $\left.\varphi_{\chi}\right|_{B}$-biprojective \\ (ii) $K$ is compact}

Proof. Suppose that $B$ is left $\left.\varphi_{\chi}\right|_{B}$-biprojective. Applying Proposition 1 in [18], $B$ is left $\left.\varphi_{\chi}\right|_{B}$-contractible. By Proposition 2.5 in $[1], L^{1}(K)$ is left $\varphi_{\chi}$-contractible. By similar argument as in the proof of Theorem $1, K$ is compact.

Conversely, let $K$ be compact with the normalized Haar measure $\omega_{K}$ (page 40 in [3]). Then, for each $\chi \in \Upsilon_{b}(K)$, we have

$$
\int_{K}|\chi(x)| \mathrm{d} \omega_{K}(x)=\int_{K} \lambda \mathrm{d} \omega_{K}(x)=\lambda|\omega(K)|=\lambda \infty,
$$

for some $\lambda>0$. So, $\chi \in L^{1}(K)$. Put $\mathbf{m}=\left(\chi / \lambda^{2}\right)$. We claim that $f * \mathbf{m}=\varphi_{\chi}(f) \mathbf{m}$ and $\varphi_{\chi}(\mathbf{m})=1$ for every $f \in L^{1}(K)$. To see this,

$$
\begin{aligned}
f * \chi(x) & =\int_{K} f(y) \chi(\bar{y} * x) d \omega_{K}(y) \\
& =\int_{K} f(y) \bar{\chi}(y) \chi(x) d \omega_{K}(y)=\varphi_{\chi}(f) \chi(x),
\end{aligned}
$$

and also 


$$
\begin{aligned}
\varphi_{\chi}(\chi) & =\int_{K} \bar{\chi}(x) \chi(x) \mathrm{d} \omega_{K}(x)=\int_{K}|\chi(x)|^{2} \mathrm{~d} \omega_{K}(x) \\
& =\lambda^{2}|\omega(K)|=\lambda^{2} .
\end{aligned}
$$

So, $L^{1}(K)$ is left $\varphi_{\chi}$-contractible. Using Proposition 2.5 in [1] again, $B$ is left $\left.\varphi_{\chi}\right|_{B}$-contractible. Hence, $B$ is left $\left.\varphi_{\chi}\right|_{B}$-biprojective (Lemma 1 in [18]).

\section{Approximate Biflatness and Left $\varphi$-Biflatness of Hypergroup Algebras}

In this section, we study approximate biflatness and left $\varphi$-biflatness of some algebras related to a hypergroup.

We remind that a Banach algebra $A$ is called approximately biflat if there exists a net $\left(\rho_{\alpha}\right)$ of $A$-bimodule morphisms from $\left(A^{\wedge} \otimes A\right)^{*}$ into $A^{*}$ such that $\rho_{\alpha}{ }^{\circ} \pi_{\mathrm{A}}^{*} \longrightarrow{ }^{\mathrm{W}^{*}} \mathrm{OT} \mathrm{id}_{\mathrm{A}^{*}}$. Here, $W^{*} \mathrm{OT}$ stands for the weak * operator topology (see [20]).

Following [21], a locally compact hypergroup $K$ is called left amenable if there exists a left invariant mean on $L U C(K)$. That is, a bounded linear functional $\mu: \operatorname{LUC}(K) \longrightarrow \mathbb{C}$ such that $\mu(1)=\|\mu\|=1$ and $\mu\left(l_{x} f\right)=$ $\mu(f)$ for all $f \in L U C(K)$ and $x \in K$.

Proposition 2. Let $K$ be a locally compact hypergroup and let $B$ be an abstract Segal algebra with respect to $L^{1}(K)$ which possess a left approximate identity. If $B$ is approximately biflat, then $K$ is left amenable.

Proof. Suppose that $B$ is approximately biflat and fix a character $\varphi \in \Delta(B)$. Since $B$ has a left approximate identity, by Proposition 2. 4 in [22], $B$ is left $\varphi$-amenable. Now, Proposition 2.3 in [1] follows that $L^{1}(K)$ is left $\varphi$-amenable. Applying Theorem 3.5 in [14], we conclude that $K$ is left amenable.

Corollary 2. Let $K$ be a locally compact hypergroup and let $B$ be an abstract Segal algebra with respect to $L^{1}(K)$ which possess a left approximate identity. If $B^{* *}$ is approximately biflat, then $\mathrm{K}$ is left amenable.

Proof. Fix a character $\varphi \in \Delta\left(L^{1}(K)\right)$. Since $B$ has a left approximate identity, similar to the arguments as in the proof of (Theorem 2.2 in [23]), approximately biflatness of $B^{* *}$ follows that $B$ is left $\left.\varphi\right|_{B}$-amenable. It deduces that $L^{1}(K)$ is left $\varphi$-amenable. Thus, by Theorem 3.5 in [14], $K$ is left amenable.

Let $A$ be a Banach algebra and $\varphi \in \Delta(A)$. Then, $A$ is called left $\varphi$-biflat if there exists a bounded linear map $\rho: A \longrightarrow\left(A^{\wedge} \otimes A\right)^{* *}$ such that

$$
\begin{aligned}
\rho(a b) & =a \cdot \rho(b)=\varphi(b) \rho(a), \\
\widetilde{\varphi}^{\circ} \pi_{\mathrm{A}}^{* *} \rho(\mathrm{a}) & =\varphi(a), \quad(a, b \in A) .
\end{aligned}
$$

Here, $\widetilde{\varphi}: A^{* *} \longrightarrow \mathbb{C}$ is a unique extension of $\phi$ which is defined by $\widetilde{\varphi}(F) F(\varphi)$ for all $F \in A^{* *}$. The right case can be defined similarly. For further information, see [24].
Theorem 3. Let $K$ be a locally compact hypergroup, $\varphi_{\chi} \in \Delta$ $\left(L^{1}(K)\right)$, and let $B$ be an abstract Segal algebra with respect to $L^{1}(K)$ which possess a left approximate identity. The following statements are equivalent:

(i) $B$ is left $\varphi$-biflat

(ii) $K$ is left amenable

(iii) $B^{* *}$ is left $\varphi$-biflat

Proof. (i) $\Rightarrow$ (ii) Let $B$ be left $\varphi$-biflat. Since $B$ has a left approximate identity, by Lemma 2.1 in [21], $B$ is left $\varphi$-amenable. It follows that $L^{1}(K)$ is left $\varphi$-amenable. Now, applying Theorem 3.5 in [14], we conclude that $K$ is left amenable.

(ii) $\Rightarrow$ (iii) Let $K$ be a left amenable hypergroup. Then, by Theorem 3.5 in [14], $L^{1}(K)$ is left $\varphi$-amenable. Now, by Proposition 2.3 in [1], $B$ is left $\varphi$-amenable. Using Proposition 3.4 in [13], $B^{* *}$ is left $\varphi$-amenable and then Lemma 2.3 in [25] implies that $B^{* *}$ is left $\varphi$-biflat.

(iii) $\Rightarrow$ (i) Suppose that $B^{* *}$ is left $\varphi$-biflat. Then, by Theorem 2.2 in [21], $B$ is left $\varphi$-biflat.

Suppose that $1 \leq p<\infty$ and $K$ is a locally compact hypergroup. Set $S_{p}(K)=L^{1}(K) \cap L^{p}(K)$ with the norm

$$
\|f\|_{S}=\|f\|_{1}+\|f\|_{p} .
$$

$S_{p}(K)$ becomes an abstract Segal algebra with respect to $L^{1}(K)$. It is known that $S_{p}(K)$ possess a left approximate identity (see [2]).

The following corollary is an easy consequence of Proposition 2 and Corollary 2.

Corollary 3. Let $K$ be a locally compact hypergroup and $1 \leq p<\infty$. If $S_{p}(K)^{* *}\left(\right.$ or $\left.S_{p}(K)\right)$ is approximately biflat, then $K$ is left amenable.

Example 1. In this example, we give a Banach algebra which is left $\varphi$-biflat but it is not right $\varphi$-biflat.

Let $A$ be a Banach space with $\operatorname{dim} A>1$ and $\varphi \in A^{*}$ be a nonzero functional such that $\|\varphi\|=1$. Define a multiplication in $A$ via $a * b=\varphi(a) b$, for all $a, b \in A$. It is easy to see that $A$ is a Banach algebra and $\Delta(A)=\{\varphi\}$. We denote the unitization of $A$ with $A^{\sharp}$. It is known that $A$ is a closed ideal in $A^{\sharp}$. Moreover, $\varphi$ has an extension to $A^{\sharp}$, that is, $\varphi_{e}: A^{\sharp} \longrightarrow \mathbb{C}$ which is given by $\varphi_{e}(a+\lambda e)=\varphi(a)+\lambda$ for all $a \in A$ and $\lambda \in \mathbb{C}$. We claim that $A^{\sharp}$ is left $\varphi_{e}$-biflat but it is not right $\varphi_{e}$-biflat. To see this, we know that $A$ is left $\varphi$-amenable, applying Lemma 3.2 in [13], it follows that $A^{\sharp}$ is left $\varphi_{e}$-amenable. It gives that $A^{\sharp}$ is left $\varphi_{e}$-biflat. Now, assume conversely that $A^{\sharp}$ is right $\varphi_{e}$-biflat. Since $A^{\sharp}$ is unital, $\left(A^{\sharp}\right)^{* *}$ has an element $m=\rho(e)$ such that $m a=\varphi(a) m$ and $\widetilde{\varphi}^{\circ} \pi_{\mathrm{A}_{\mathrm{e}}}^{* *}(\mathrm{~m})=1$, for all $a \in A$. Following the similar arguments as in Theorem 1.4 in [13], we have a bounded net $\left(m_{\alpha}\right)$ in $A_{e}$ such that $m_{\alpha} a-\varphi(a) m_{\alpha} \longrightarrow 0$ and $\varphi\left(m_{\alpha}\right)=1$, for all $a \in A_{e}$. Pick an element $a_{0}$ in $A$ such that $\varphi\left(a_{0}\right)=1$. Replace $\left(m_{\alpha}\right)$ with $\left(a_{0} m_{\alpha}\right)$, we may suppose that $\left(m_{\alpha}\right)$ is a bounded net in $A$ such that $m_{\alpha} a-\varphi(a) m_{\alpha} \longrightarrow 0$ and $\varphi\left(m_{\alpha}\right)=1$, for all $a \in A$. However, 


$$
1 a-\varphi(a) m_{\alpha}=\varphi\left(m_{\alpha}\right) a-\varphi(a) m_{\alpha}=a m_{\alpha}-\varphi(a) m_{\alpha} \longrightarrow 0,
$$

for all $a \in A$. Let $a$ be any element in $\operatorname{ker} \varphi$ and put it at above fact. It follows that $\operatorname{ker} \varphi=(0)$. So, $\varphi$ is an isomorphism. Therefore, $\operatorname{dim} A=\operatorname{dim} \mathbb{C}=1$ which is a contradiction.

\section{Data Availability}

The data that support the findings of this study are available from all the authors.

\section{Conflicts of Interest}

The authors declare that there are no conflicts of interest regarding the publication of this paper.

\section{Acknowledgments}

The first author is thankful to Ilam University, for their support.

\section{References}

[1] C. F. Dunkl, "The measure algebra of a locally compact hypergroup," Transactions of the American Mathematical Society, vol. 179, pp. 331-348, 1973.

[2] R. I. Jewett, "Spaces with an abstract convolution of measures," Advances in Mathematics, vol. 18, no. 1, pp. 1-101, 1975.

[3] R. Spector, "Mesures invariantes sur les hypergroupes," Transactions of the American Mathematical Society, vol. 239, pp. 147-165, 1978.

[4] A. Y. Helemskii, The Homology of Banach and Topological Algebras, Kluwer Academic Publishers, Holland, The Netherlands, 1989.

[5] H. Rahimi, M. Ghahramani, and S. Moayeri, "Biprojectivity and weak amenability of some Banach algebras," Contemporary Mathematics, vol. 547, pp. 207-216, 2010.

[6] M. Essmaili, A. R. Medghalchi, and R. Ramezani, “ $\varphi$ \$\$ varphi $\$ \$ \varphi$-biprojectivity of Banach algebras with applications to hypergroup Algebras," Bulletin of the Iranian Mathematical Society, vol. 45, no. 2, pp. 359-376, 2019.

[7] Y. Zhang, "Nilpotent ideals in a class of Banach algebras," Proceedings of the American Mathematical Society, vol. 127, no. 11, pp. 3237-3242, 1999.

[8] A. Sahami and A. Pourabbas, "Approximate biprojectivity of certain semigroup algebras,” Semigroup Forum, vol. 92, no. 2, pp. 474-485, 2016.

[9] E. Kaniuth, A. T. Lau, and J. Pym, "On $\phi$-amenability of Banach algebras," Mathematical Proceedings of the Cambridge Philosophical Society, vol. 144, no. 1, pp. 85-96, 2008.

[10] B. E. Johnson, "Cohomology in Banach algebras," Memoirs of the American Mathematical Society, vol. 127, 1972.

[11] Z. Hu, M. Sangani Monfared, and T. Traynor, "On character amenable Banach algebras,” Studia Mathematica, vol. 193, no. 1, pp. 53-78, 2009.

[12] R. Nasr-Isfahani and S. Soltani Renani, "Character contractibility of Banach algebras and homological properties of Banach modules," Studia Mathematica, vol. 202, no. 3, pp. 205-225, 2011.
[13] A. Sahami and M. Rostami, "Some cohomological notions in Banach algebras based on maximal ideal space," Iranian Journal of Science and Technology, vol. 6, 2021.

[14] W. R. Bloom and H. Heyer, Harmonic Analysis of Probability Measures on Hypergroups, Walter de Gruyter, Berlin, Germany, 1995.

[15] Y. Chapovsky, "Existence of an invariant measure on a locally compact hypergroup," 2012, http://arxiv.org/abs/1212.6571.

[16] F. Ghahramani and A. T. M. Lau, "Weak amenability of certain classes of Banach algebras without bounded approximate identities," Mathematical Proceedings of the Cambridge Philosophical Society, vol. 133, no. 2, pp. 357-371, 2002.

[17] M. Alaghmandan, R. Nasr-isfahani, and M. Nemati, "Character amenability and contractibility of abstract Segal algebras," Bulletin of the Australian Mathematical Society, vol. 82, no. 2, pp. 274-281, 2010.

[18] H. Javanshiri and M. Nemati, "Invariant $\varphi$-means for abstract Segal algebras related to locally compact groups," Bulletin of the Belgian Mathematical Society Simon Stevin, vol. 25, pp. 687-698, 2018.

[19] H. Samea, "Essential amenability of abstract Segal algebras," Bulletin of the Australian Mathematical Society, vol. 79, no. 2, pp. 319-325, 2009.

[20] E. Samei, N. Spronk, and R. Stokke, "Biflatness and pseudoamenability of Segal algebras," Canadian Journal of Mathematics, vol. 62, no. 4, pp. 845-869, 2010.

[21] J. Laali and R. Ramezani, "Approximate amenability and contractibility of hypergroup algebras," Annals of Functional Analysis, vol. 9, no. 4, pp. 551-565, 2018.

[22] M. Nemati, "Some homological properties of Banach algebras associated with locally compact groups," Colloquium Mathematicum, vol. 139, no. 2, pp. 259-271, 2015.

[23] A. Sahami, "On biflatness and $\varphi$-biflatness of some Banach algebras," Bucharest Scientific Bulletin-Series A-Applied Mathematics and Physics Metrics, vol. 80, no. 1, pp. 111-122, 2018.

[24] A. Sahami, M. Rostami, and A. Pourabbas, "On left $\varphi$-biflat Banach algebras," Commentationes Mathematicae Universitatis Carolinae, vol. 63, pp. 337-344, 2020.

[25] S. S. Baladezaei, A. Mahmoodi, A. Sahami, and M. Rostami, "Left $\varphi$-biflatness and $\varphi$-biprojectivity of certain banach algebras with applications," 2019, http://arxiv.org/abs/1811. 02393. 